\title{
Animal Symposia and Workshops
}

Published online: 6 May 2021

\section{A-1}

Ephydatia muelleri - a Sponge Model for Evolutionary Biology and Beyond. SCOTT ANTHONY NICHOLS. Department of Biological Sciences, University of Denver, CO. Email: scott.nichols@du.edu

Sponges have key significance for the study of animal evolution. They are $>600 \mathrm{My}$ divergent from all other animals and are no more closely related to other invertebrates than to vertebrates. They have fewer than 20 cell types, and lack fundamental traits shared by other animals, such as neurons, muscles, axes of symmetry and polarity, and a gut - indeed, they are the only animals to retain the ancestral mode of animal nutrition, direct phagocytosis of bacteria. Beyond evolutionary biology, sponges have increasingly recognized ecological significance in declining coral reef habitats, complex microbial associations and related secondary-metabolite chemistris with proven pharmaceutical applications, and they exhibit the unparalleled capacity to regenerate their entire body from mixtures of dissociated cells. A primary bottleneck that limits mechanistic research in sponges is the lack of widely available model that can be cultivated in the laboratory and is amenable to genetic manipulation. To address this gap, we are working to develop genetic and biochemical techniques to study the freshwater species Ephydatia muelleri. E. muelleri is attractive because a chromosome-level genome assembly is available, a single-cell RNAseq atlas is underway, and because it can be grown axenically in the laboratory from overwintering spores (gemmules) that contain adult stem cells in a state of diapause. I will provide an overview of the biology of E. muelleri, highlight experimental techniques currently available for its study, and will discuss ongoing efforts to develop transfection and viral transduction methods for genetic manipulation.

\section{A-2}

In Vitro Cell Culture: A Powerful Tool for CnidarianDinoflagellate Symbiosis Studies. S. BARNAY-

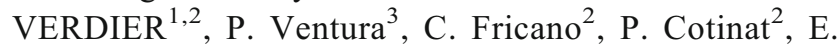

Röttinger ${ }^{2}$, and P. Furla ${ }^{2}$. ${ }^{1}$ Sorbonne Université, UFR 927, 4 place Jussieu, F-75252 Paris, FRANCE; ${ }^{2}$ Université Côte d'Azur, CNRS, INSERM, Institute for Research on Cancer and Aging (IRCAN), 28 avenue de Valombrose, F-06107 Nice, FRANCE; and ${ }^{3}$ THALASSA Marine Research \& Environmental Awareness Company, F-06000 Nice, FRANCE. Email: stephanie.barnay-verdier@upmc.fr

The development of mammalian cell cultures had an enormous impact on scientific discoveries. We anticipate that in vitro cell culture will create new opportunities to study Cnidarian-Dinoflagellate symbiosis. This mutualistic intracellular association is one of the most important marine symbioses. Inside the cytoplasm of host gastrodermal cells, the symbiont triggers a fine communication with the animal cells. Although this symbiosis has been studied for years, the molecular dialog between the two partners and the symbiosis properties of the animal cells remain unresolved due to the absence of a reliable model for cellular approaches. To address this knowledge gap, we developed an in vitro Cnidarian primary cell culture from the symbiotic sea anemone, Anemonia viridis. More particularly, we established and maintained a gastrodermal primary cell culture characterized by a specific architecture (i.e. cell aggregates), a high viability (90\%), and a constant cell growth during several weeks. In addition, we succeeded to design a cryopreservation procedure. This protocol enabled us to obtain high cell survival after thawing (70\%) and a full long-term recovery of the cell culture behavior. This powerful tool allowed to lead cellular functional assays in order to address the intrinsic capacity of animal cells to deal with environmental perturbations, such as hyperthermia, involved in symbiosis breakdown. Gastrodermal primary cell cultures were submitted to hyperthermal stress $(+5$ and $+8^{\circ} \mathrm{C}$ ). Though cell viability was not affected, cell growth drastically decreased. In addition, $a+8^{\circ} \mathrm{C}$ hyperthermia induced a transient increase of antioxidant defenses but no protein damages. These results demonstrated an intrinsic resistance of cnidarian gastrodermal cells and then confirmed the role of symbionts in the hyperthermia sensitivity leading to bleaching. Taken together these results allow us to propose A. 
viridis gastrodermal primary cell culture as new model for studies on cellular processes involved in CnidarianDinoflagellate symbiosis and in environmental stress responses.

\section{A-3}

Adult Stem Cells from Marine Invertebrates as Building Blocks for Immortal Cell Cultures. B. RINKEVICH. National Institute of Oceanography, Haifa, ISRAEL. Email: buki@ocean.org.il

Despite several decades of extensive research efforts, there is yet no single permanent cell line available from marine invertebrates as these cells stop dividing in vitro within $24-72 \mathrm{~h}$ after their isolation, starting cellular quiescence. This ubiquitous quiescent state should be modified in a way that at least some of the quiescent cells will become pluripotent, so they will have the ability to divide and become immortal. Following the above need, a decade ago a suggestion has been put forward to use adult stem cells (ASCs) from marine organisms. Many marine invertebrate taxa maintain large pools of ASCs that emerge at different lifespan periods/events, presenting both differentiated and undifferentiated phenotypes, constitute up to $40 \%$ of animal cells, are generally pluri/ totipotent cells, often with no germ-line sequestering. Many of these ASCs express germ-cell markers, appear not to reside in discrete niches, participate in whole-body regeneration, torpor, agametic asexual-reproduction, indeterminate-growth and are considered as legitimate units of selection. These ASCs are actively proliferating in vivo, resembling the vertebrates' cancer stem cells features. The employment of these cells carries the potential to revolutionize the discipline of marine invertebrate cell cultures. The talk will further discuss the unique features associated with the marine invertebrates ASCs.

\section{A-4}

In Vitro Cell-based Systems to Advance Functional Genomic Studies in Echinoderms. ANDREA BODNAR and Amanda Baryshyan. Gloucester Marine Genomics Institute, Gloucester, MA. Email: andrea.bodnar@gmgi.org

For more than a century, sea urchins and other echinoderms have been paradigmatic for understanding many biological processes such as cell cycle regulation, the respiratory burst, egg activation, developmental gene regulatory networks, biomineralization, innate immunity, tissue regeneration, and the evolution of major body plans. Remarkably, these important advancements were made without the benefit of genetic tools that enable controllable or heritable targeted gene alterations or cell lines for in vitro applications. The availability of primary and established cell lines from echinoderms would provide a valuable resource for mechanistic studies of gene function and regulation, and enable new avenues of investigation that fully exploit the important properties of echinoderms as a research model. Primary cell cultures derived from sea urchin embryos and adult tissues have been used to evaluate DNA repair, biomineralization, and to study the production of naphthoquinone pigments. However, these cells have had limited proliferative capacity and no continuously dividing cell line has yet been established for echinoderms. With a focus on sea urchin embryonic and adult stem cells, the aim of our work is to use a combination of "omics" technologies to derive optimal growth conditions and to develop CRISPR gene editing tools for targeted genetic manipulation of cultured cells. Using a panel of stem cell markers, we have identified putative stem cells in adult sea urchin tissues and have derived primary cells from both embryos and adult tissues. Metabolomics and proteomics analyses of coelomic fluid are being used to inform media design, and transfection conditions are being optimized for delivery of CRISPR reagents targeting selected tumor suppressor genes. Cell culture tools will add a new era of discovery to the remarkable history of the sea urchin enabling mechanistic investigation of fundamental and integrated biological processes in cell biology, development, immunity, tissue regeneration, and beyond.

\section{A-5}

Investigating Enterotropic Virus Infections in Human Intestinal Organoids. CHRISTIANE E. WOBUS. University of Michigan Medical School, 1150 West Medical Center Dr., Ann Arbor, MI. Email: cwobus@umich.edu

The gastrointestinal mucosa is organized into villi and crypts and comprised of a layer of epithelial cells, the lamina propria containing immune cells and a layer of smooth muscle cells. Recent developments in biomimetic human models of enteric disease are opening new possibilities for studying humanspecific host-microbe interactions. Wide application of these models hold promise for revealing new knowledge about the complex interplay between pathogen, host, and commensals and the development of effective disease prevention and control measures for enteric diseases. Human intestinal organoids are derived from stem cells isolated from intestinal biopsy tissues or from induced pluripotent stem cells. Most laboratories use human intestinal enteroids (HIE), which are epithelium-only intestinal organoids derived biopsy-derived stem cells. HIE have been successfully used to culture a range of enterotropic pathogens, including human norovirus and human rotavirus. We recently established HIE as a culture system for human astroviruses from all clades and demonstrated its superiority over traditional transformed intestinal cell lines to study innate immune signaling. HIEs also support 
robust SARS-CoV-2 infection and can be used as a validation platform for antiviral drug testing. However, a key limitation for studying virus-host interactions using the current epithelium-only HIE system is the lack of immune cells and microbes, which are important players in intestinal homeostasis and critical drivers for enteric diseases. Thus, efforts are underway in the field for further innovations to improve these models and enhance their complexity.

\section{A-6}

Living the Sweetest Life - A Preferential Recognition of SARS-CoV-2 to 3-O Sulfated Heparan Sulfate. VAIBHAV TIWARI ${ }^{1}$, Ritesh Tandon ${ }^{2}$, Nehru Viji Sankaranarayanan $^{3}$, Jacob C. Beer ${ }^{1}$, Ellen K. Kohlmeir ${ }^{4}$, Michelle Swanson-Mungerson ${ }^{1}$, and Umesh R. Desai ${ }^{3}$. ${ }^{1}$ Department of Microbiology and Immunology, Midwestern University, Downers Grove, IL 60515; ${ }^{2}$ Department of Microbiology and Immunology, University of Mississippi Medical Center, Jackson, MS 39216; ${ }^{3}$ Department of Medicinal Chemistry and Institute for Structural Biology, Drug Discovery and Development, Virginia Commonwealth University, 800 E. Leigh Street, Suite 212, Richmond, VA 23219; and ${ }^{4}$ Core Facility, Midwestern University, Downers Grove, IL60515. Email: vtiwar@midwestern.edu, urdesai@vcu.edu

A novel severe acute respiratory syndrome (SARS)-like coronavirus (SARS-CoV-2) is causing the global coronavirus disease 2019 (COVID-19) pandemic. Understanding how SARS-CoV-2 enters human cells is a high priority to prevent infection. Using reporter-based cell fusion assay, we demonstrate heparan sulfate proteoglycans (HSPGs) modified by the 3-O-sulfotransferase isoform-3, but not isoform-5, preferentially increased SARS CoV-2 spike glycoprotein $(\mathrm{SgP})$ mediated cell-to-cell fusion in comparison to control, unmodified, wild-type HSPGs. Interestingly the event of virus-cell fusion was mediated in absence of human ACE-2 - a previously reported receptor for SARS-CoV-2 cell entry. In addition, the computational studies support preferential recognition of the receptor-binding domain of $\mathrm{SgP}$ by 3-O-sulfated HS sequences. Competition with either fondaparinux, a 3-Osulfated HS-binding oligopeptide, or a synthetic, nonsugar small molecule, blocked SgP-mediated cell-to-cell fusion. Finally, the synthetic, sulfated molecule inhibited fusion of GFP-tagged pseudo-SARS-CoV-2 with human 293 T cells with sub-micromolar potency. Overall, overexpression of 3-O-sulfated HSPGs contribute to fusion of SARS-CoV-2, which could be effectively antagonized by a synthetic, small molecule. Taken together the study provides insight into intervention strategies that target SARS-CoV-2 cell entry.

\section{A-7}

Hello Gorgeous, You're Just My Type: Using Pseudotyping Technology to Elucidate HSV-1 Entry. EKATERINA E. HELDWEIN $^{1,2}$, Adam T. Hilterbrand ${ }^{1}$, and Raecliffe E. Daly ${ }^{1,2}$. ${ }^{1}$ Department of Molecular Biology and Microbiology, Tufts University School of Medicine, Boston, MA 02111 and ${ }^{2}$ Graduate Program in Cellular, Molecular, and Developmental Biology, Tufts University School of Medicine, Boston, MA 02111. Email: katya.heldwein@tufts.edu

Herpes simplex viruses (HSV-1 and HSV-2) contain up to 16 different proteins in their envelopes. Four of these, glycoproteins $\mathrm{gB}, \mathrm{gD}, \mathrm{gH}$, and $\mathrm{gL}$, are termed essential with regard to entry whereas the rest are typically referred to as non-essential based on the entry phenotypes of the respective single genetic deletions. However, the single-gene deletion approach, which relies on robust loss-of-function phenotypes, may be confounded by functional redundancies among the many HSV1 envelope proteins. We have developed a pseudotyping platform, in which the essential four entry glycoproteins are isolated from the rest, which can be added back individually for systematic gain-offunction entry experiments. Here, we show the utility of this platform for dissecting the contributions of HSV envelope proteins, both the essential four and the remaining dozen (using $\mathrm{gC}$ as an example), to HSV entry.

\section{A-8}

Organoid Modeling the Development and Stem Cells of the Upper GI Tract. JIANWEN QUE. Columbia University, Department of Medicine, BB810, 650 West 168th Street, New York, NY 07024. Email: jq2240@ cumc.columbia.edu

Study of animal models has provided initial insights into the molecular mechanisms regulating the morphogenesis and stem cells of the upper gastrointestinal (GI) tract. With the use of organoid models this knowledge can now be translated into human GI study. Our laboratory combines mouse genetics model and human pluripotent stem cell differentiation to study how the esophagus is formed at the embryonic stage and how this tubular organ is maintained in adults. Our studies revealed that multiple signaling pathways including BMP, HIPPO and NOTCH play essential roles in the development and stem cell maintenance in the upper GI tract (Bailey et al., Nature. 2017 Oct 26;550(7677):529-533; Jiang et al., Zhang et al., Cell Stem Cell. 2018 Oct 4;23(4):516-529.e5; Jiang et al., Nature. 2017 Oct 26;550(7677):529-533.) 


\section{A-12}

Visualizing Cell Competition and Mechanisms of Occult Tumorigenesis Ex Vivo. Joshua D. Ginzel ${ }^{1,2}$, Chaitanya R. Acharya $^{1}$, Ping Wang ${ }^{1}$, Alexis Schmid ${ }^{1}$, Peter G. Boone ${ }^{1,2}$, Lauren K. Rochelle ${ }^{2}$, Wendy Roberts ${ }^{2}$, Lawrence S. Barak ${ }^{2}$, Marc G. Caron ${ }^{2}$, Robert D. Cardiff ${ }^{3}$, Alexander D. Borowsky $^{3}$, H. Kim Lyerly ${ }^{1,4}$, and JOSHUA C. SNYDER $^{1,2}$. ${ }^{1}$ Department of Surgery, Division of Surgical Sciences, Duke University Medical Center, Box 2606, Durham, NC 27710; ${ }^{2}$ Department of Cell Biology, Duke University Medical Center, Durham, NC; ${ }^{3}$ Department of Pathology and Laboratory Medicine and The Center for Comparative Medicine, University of California-Davis, Davis, CA; and ${ }^{4}$ Department of Immunology, Duke University School of Medicine, Durham, NC 27710. Email: joshua.snyder@duke.edu

Cell competition in the intestinal stem cell niche is a stochastic process controlling stem cell number and tissue homeostasis. Mutations to protooncogenes and tumor suppressors are thought to bias competition and lead to malignant clonal expansion that progressively advance to cancer. Here we utilize a cancer rainbow modeling system to incite multiple oncogenes and then lineage trace their fitness effects in vivo and ex vivo. We show that the microenvironment and biophysical constraints of the intestine are both critical suppressive forces that prevent deterministic oncogenic behaviors in the stem cell pool. Pharmacological and CRISPR/Cas9 perturbations of LGR5 signaling revealed how extrinsic signaling nodes control this stochastic behavior. Deprivation of the environmental cues increased intestinal stem cell turnover and resulted in more rapid fixation of stem cell clones in the crypt. These data demonstrate that drugs targeting LGR5 could be used to disrupt stem cell homeostasis and potentially deplete colorectal cancer stem cells.

\section{A-13}

Modeling Neuropsychiatric Disorders Using Human Induced Pluripotent Stem Cell Derived Brain Organoids. ANNIE KATHURIA $^{1,2,3,7}$, Kara Lopez-Lengowski ${ }^{1,2}$, and Rakesh Karmacharya ${ }^{1,2,3,4,5,6,7}$. ${ }^{1}$ Center for Genomic Medicine, Massachusetts General Hospital, Boston, MA; ${ }^{2}$ Chemical Biology Program, Broad Institute of MIT \& Harvard, Cambridge, MA; ${ }^{3}$ Department of Psychiatry, Harvard Medical School, Boston, MA; ${ }^{4}$ Schizophrenia and Bipolar Disorder Program, McLean Hospital, Belmont, MA; ${ }^{5}$ Program in Neuroscience, Harvard University, Cambridge, MA; ${ }^{6}$ Program in Chemical Biology, Harvard University, Cambridge, MA; and ${ }^{7}$ Harvard Stem Cell Institute, Cambridge, MA. Email: akathuria@mgh.harvard.edu
Investigations focused on the biology of psychiatric disorders present significant scientific and methodological challenges. A primary barrier to scientific investigations of psychiatric disorders has been the infeasibility of accessing live neuronal tissue from patients to undertake experiments in the laboratory. The discovery of a way to reprogram somatic cells to pluripotency using defined factors have opened new avenues for the study of disorders affecting the human brain. In these studies, we have determined genetic and functional profiles of mini brains generated from patients suffering neuropsychiatric disorders. The mini brains generated from schizophrenia subjects show differences in the pattern of expression of genes involved in synaptic biology, neurodevelopment and immune response as well as genes involved in mitochondrial function and modulation of excitation/inhibition balance. The mini brains show specific deficits in mitochondrial physiology and a diminished response to stimulation and depolarization. In conclusion, we provide the first comprehensive studies of comparing mini brains generated from schizophrenia subjects and compared it to healthy subjects. We delineate transcriptomic differences in specific pathways, including down-regulation of genes involved in cell adhesion, neurodevelopment and synaptic biology in both disorders along with up-regulation of genes involved in immune signaling.

\section{A-15}

Emodin Attenuates Pathological Cardia Remodeling: Is There a Role for the Microbiome? Levi Evans ${ }^{1,2}$, Tori Shock $^{3}$, Nathaniel Hubert ${ }^{3}$, Julia Moore, ${ }^{3}$ Y Yqui Shen ${ }^{1}$, Maheshi Athukorala ${ }^{1}$, Kristina Martinez-Guryn ${ }^{3}$, and BRADLEY S FERGUSON ${ }^{1,2,4}$. ${ }^{1}$ Department of Nutrition, University of Nevada, Reno, Reno, NV 89557 ; ${ }^{2}$ Environmental Sciences Program, University of Nevada, Reno, Reno, NV 89557; ${ }^{3}$ Biomedical Sciences Program, Midwestern University, Downers Grove, IL. 60515; and ${ }^{4}$ Center of Biomedical Research Excellence for Molecular and Cellular Signal Transduction in the Cardiovascular System, University of Nevada, Reno, NV 89557. Email: bferguson@unr.edu

Evidence suggests that food bioactives, or phytochemicals, prevent pathological cardiac hypertrophy through regulation of gene expression, inflammation and oxidative stress. Recently, we tested the postulate that emodin, an athraquinone found in rhubarb, buckthorn, knotweed, beans and cabbage, could protect the heart via transcriptome-wide changes in gene expression. Indeed, we reported that emodin normalized global changes in gene expression in stress-induced cardiac myocytes and attenuated pathological cardiac hypertrophy and fibrosis in hypertensive male and female mice. These data suggest that emodin directly targets the 
heart. However, recent reports have shown that little of the parent compound emodin makes it to the heart following oral consumption. These new findings therefore, implicate emodin-derived microbial metabolites as critical for cardioprotection. Because the microbiome has emerged as a key player in diet-related diseases including cardiovascular disease (CVD), we further examined the effects of emodin on the microbiome of hypertensive and healthy mice. Here, we report several novel and interesting observations: 1) hypertension decreased microbial diversity; 2) emodin also decreased bacterial diversity in healthy mice by day 3 of ingestion, yet increased the abundance of beneficial bacteria (e.g. Bacteroides thetaiotamicron); 3) emodin normalized the microbiome in hypertensive animals similar to control mice and improved bacterial composition that favored leanness (e.g. Roseburia; Akkermansia) and cardioprotection (e.g. Akkermansia); and 4) microbial composition was dependent on the route for emodin administration in which gavage-dosed animals displayed an increase in selective protective bacteria (e.g. Roseburia), while intraperitoneal-dosed mice exhibited an increase in other protective bacterial species (e.g. Bacteroides thetaiotamicron) suggesting that bacterial and liver metabolism of emodin is important for cardioprotection. While correlative, these findings suggest that the cardioprotective actions of emodin are driven, in part, through the regulation of the microbiome. Current analysis of the metabolome and lipidome are underway as are fecal microbiota transplant studies to further elucidate the role for the microbiome and microbial-derived emodin metabolites in cardio-protection.

\section{A-16}

Individual Variation in Prebiotic Response. L. A. DAVID ${ }^{1,2}$, Zachary C. Holmes ${ }^{1}$, Max M. Villa ${ }^{1,2}$, Heather K. Durand ${ }^{1,2}$, Sharon Jiang ${ }^{1,2}$, Eric P. Dallow ${ }^{1,2}$, Brianna L. Petrone ${ }^{1,3}$, Justin D. Silverman ${ }^{4,5,6}$, and Pao-Hwa $\operatorname{Lin}^{7,8}$. ${ }^{1}$ Department of Molecular Genetics and Microbiology, Duke University, Durham, NC; ${ }^{2}$ Center for Genomic and Computational Biology, Duke University, Durham, NC; ${ }^{3}$ Medical Scientist Training Program, Duke University, Durham, NC; ${ }^{4}$ College of Information Science and Technology, Penn State University, University Park, PA; ${ }^{5}$ Department of Medicine, Penn State University, Hershey, PA; ${ }^{6}$ Institute for Computational and Data Science, Penn State University, University Park, PA; ${ }^{7}$ Duke Molecular Physiology Institute, Duke University, Durham, NC; and ${ }^{8}$ Duke Cancer Institute, Duke University, Durham, NC. Email: lawrence.david@duke.edu

Short-chain fatty acids (SCFAs) derived from gut bacteria are associated with protective roles in diseases ranging from obesity to colorectal cancers. Intake of different microbially accessible dietary fibers (prebiotics) lead to varying effects on gut bacterial SCFA production in human cohort studies, and gut microbial responses to nutritional interventions vary by individual. It is therefore possible that in order to maximize the benefit of microbiota-targeting prebiotics, these supplemental regimens should be customized to individuals. Here, we explored this nutritional strategy by conducting a fully balanced, three-way crossover study of inulin, dextrin, and galactooligosaccharides (GOS) treatment in healthy adults. We found that within individuals, prebiotic impacts on fecal butyrate levels were correlated across prebiotics. Across individuals, prebiotic response was inversely related to basal fecal SCFA concentration, which in turn, was associated with habitual intake of dietary fiber. In vitro fecal assays also supported a negative association between prebiotic metabolic potential and baseline fiber intake. Together, these findings support developing personalized prebiotic regimens that focus on selecting individuals who stand to benefit, and that such individuals are likely to be deficient in fiber intake.

\section{A-17}

Developing a Fluorescence Biosensor for the Herbicide Glyphosate. SHAHIR S. RIZK, Pierre-Emmanuel N'Guetta, and Maggie M. Fink. Indiana University South Bend, Department of Chemistry and Biochemistry, 1700 Mishawaka Ave, South Bend, IN 46615. Email: srizk@iusb.edu

Glyphosate is a phosphonate used to kill weeds by blocking pathways essential to plant growth. Currently, glyphosate is the most popular herbicide used around the globe. It has been recently classified as a probable carcinogen. It is also considered a potential environmental hazard. Several states are planning to restrict its use, while it has already been banned in the state of California. Hence, there is a need for the development of reliable detection methods for glyphosate in the soil, rivers or drinking water. This can help determine its concentration and effect on the environment. We took a protein engineering approach to develop a fluorescence-based biosensor for glyphosate. Phosphonate binding protein $(\mathrm{PhnD})$ is a bacterial protein that binds to a variety of phosphonates with different affinities. This protein allows the bacteria to sequester phosphorus from the environment. Previous work showed that the protein binds to glyphosate but with very low affinity (dissociation constant $0.65 \mathrm{mM}$ ). Guided by the crystal structure of the PhnD, we constructed a set of mutations in the binding region in order to enhance binding affinity for glyphosate. One of the mutants showed nearly $\sim 100$-fold improvement in the binding affinity, allowing detection of glyphosate in the low micromolar range. The main advantage of using $\mathrm{PhnD}$ as a biosensor is that the protein undergoes a large conformational change upon binding to glyphosate. By attaching an environmentally sensitive fluorophore, binding can be detected by monitoring changes in fluorescence. 
Furthermore, the fluorescence change is ratiometric, allowing for a standardized way to correlate fluorescence ratios with glyphosate concentrations. We believe that this biosensor can be incorporated in a device that allows real-time detection of glyphosate levels in soil or water to help with environmental monitoring and decontamination efforts.

\section{A-18}

Atomic Force Microscopy Combined with Machine Learning as a New Approach to Detect Cell Abnormalities: Towards Outeromics. I. SOKOLOV. Department of Mechanical Engineering, Department of Biomedical Engineering, and Department of Physics, Tufts University, Medford, MA 02155. Email: Igor.Sokolov@Tufts.edu

New multi-dimensional atomic force microscopy (AFM) modalities developed in our group, Ringing mode and FTNanoDMA allow collecting more than 30+ images of a cell surface simultaneously. Ringing mode presently allows for simultaneous recording of 14 maps/images of the distribution of different physical and chemical properties of the cell surface. FT-NanoDMA is capable of recording 21 images of mechanical properties of the cell surface. We have shown that even a part of this enormous amount of information can be used to identify a cell phenotype. For example, we demon- strated that it was possible to differentiate between different stages of progression of cells towards human epithelial cervical cancer. The difference between normal and cancer cells was unambiguous and the level of single cells. Furthermore, we have shown that the analysis of images of just five cells allows identifying active bladder cancer in patients under surveillance with an accuracy of $94 \%$. Recently, we have demonstrated that the method allows to segregate human epithelial colorectal cancer cells, which have a particular mutation leading to the increase of aggressiveness of cancer. The accuracy of such segregation was $90+$ and the level of single cells. This is higher than the current accuracy of DNA analysis of single cells (which is $\sim 80 \%$ ), and comparable to the methods based on the single-cell RNA analysis. Therefore, we conceive that the approach might bring into being a new "omic", which we propose to call "outeromics" similar to pretty proteomics and genomics. This wealth of information obtained with the help of new modalities requires special methods of data processing. We proposed to use machine learning to classify the obtained images. In this talk, I will describe our solution to adapt AFM imaging for use together with machine learning. The key is in a substantial reduction of the dimension of the data space by using so-called surface parameters. An example of this approach will be described. The steps to produce sufficient statistics using bootstrap methods will be overviewed. An important problem of overtraining will be discussed. 